\title{
Salivary protein glycopatterns for natural regulation of oral microbiota
}

\author{
Zheng Li ( $\nabla$ zhengli@nwu.edu.cn ) \\ Northwest University https://orcid.org/0000-0002-1839-3450 \\ Jian Shu \\ Northwest University \\ Yu Hanjie \\ Northwest University \\ Yan Wang \\ Northwest University \\ Xiameng Ren \\ Northwest University \\ Kun Zhang \\ Northwest University \\ Zhen Tang \\ Northwest University \\ Wentian Chen \\ Northwest University \\ Baozhen Li \\ The First Affiliated Hospital of Xi'an Jiaotong University
}

\section{Biological Sciences - Article}

Keywords: oral microbiota, glycoconjugates, oral health

Posted Date: February 12th, 2021

DOI: https://doi.org/10.21203/rs.3.rs-221122/v1

License: (c) (1) This work is licensed under a Creative Commons Attribution 4.0 International License.

Read Full License 


\section{Abstract}

The oral microbiota is the direct precursor of dental caries and periodontitis, which are the most common microbial-induced diseases worldwide. The distinct microenvironment at the oral barrier breeds unique microbial communities, which are regulated by host factors (inflammation or dietary sugars) 1 . Increasing evidence indicates that dysbiosis of oral microbial communities is associated with many human diseases2-5. Our studies demonstrated that human disease could induce different alterations in salivary protein glycopatterns6. However, the relationship between salivary protein glycopatterns and oral microbial communities is unknown. Here, we report that altered salivary protein glycopatterns, namely, fucosylated or sialylated structures induced by gastric cancer (GC) or type 2 diabetes mellitus (T2DM), respectively, are also drivers of dysbiosis of oral microbial communities and ultimately dental caries and periodontitis. The fucosylated neoglycoproteins and sialic acid (SA) a2-3 galactose (Gal) structure can inhibit the growth or/and adhesion of Aggregatibacter segnis and Candida albicans from the oral cavity of patients with GC and T2DM, respectively. These findings provide a novel theory that dynamic communities of oral microbiota are regulated naturally by host salivary protein glycopatterns, having important implications for developing new carbohydrate drugs for oral and body health.

\section{Main Text}

The microbiota in the oral cavity plays an important role in maintaining oral health and even body health. The nososymbiocity of the polymicrobial communities involved in both dental caries and periodontitis is largely regulated by host factors, predominantly dietary sugars and inflammation. Recently, increasing evidence has shown that the oral microbiota is associated with human diseases, including diabetes, obesity, and cancer ${ }^{7,8}$. Our previous studies demonstrated that different types of diseases (e.g., gastric cancer (GC), type 2 diabetes mellitus (T2DM), hepatopathy, and breast disease) could induce various alterations in salivary protein glycopatterns (e.g., fucosylation in GC and sialylation in T2DM) ${ }^{9-12}$. Furthermore, can altered salivary protein glycopatterns also impact the dynamic and polymicrobial oral microbiome? We hypothesize that altered salivary protein glycopatterns are possible drivers leading to dysbiosis of oral microbial communities and ultimately dental caries and periodontitis.

To address this hypothesis, we first studied the changes in the oral microbiota associated with GC. Among the 24 samples, an estimated 79 thousand raw sequence reads per sample with a median length of 423 base pairs is obtained. After quality trimming and chimera checking, 75 thousand clean sequence reads per sample remained, which are recovered for downstream analysis. The detailed sequencing data of each sample are shown in Extended Data Table S1. In total, 789 unique species are identified in the oral cavity of healthy volunteers (HVs) and patients with GC; 525 species are common in the HVs and GC patients, while 128 and 136 species are observed in only the HVs and GC patients, respectively (Fig. 1A). The alterations in the microbial communities are evaluated between the HV and GC groups. The alphadiversity analysis shows that there are no significant differences between the groups (Fig. 1B-E), while the beta-diversity analysis shows that the oral microbiota profiles of the two groups are different, and can be distinguished using PCA, PCoA and NMDS plots (Fig. 1F-H). 
To further investigate the composition of oral microbial communities, the Wilcoxon test is used to compare the variance at each taxonomic level between the HV and GC groups (Table 1). The results show that there are no significant differences at the phylum, class and order levels; however, the members of Carnobacteriaceae are increased in the GC patients compared with the HVs at the family level (Fig. 2A). The proportions of the genera Alloprevotella and Megasphaera are significantly increased whereas those of Granulicatella, Bregeyella and TM7[G-6] are decreased in the GC group (Fig. 2B). Five species (Oral taxon 392, Oral taxon 308, Aggregatibacter segnis (A. segnis), Megasphaera micronuciformis (M. micronuciformis), and Oral taxon 396) are significantly increased $(p<0.05)$ and 2 (Streptococcus salivarius (S. salivarius) and Oral taxon 870$)$ are significantly decreased $(p<0.01)$ in the GC patients compared with the HVs (Fig. 2C). Furthermore, the relative abundances of $A$. segnis, M. micronuciformis and $S$. salivarius are verified by PCR and qPCR, and their results are consistent with the results obtained by $16 \mathrm{~S}$ rDNA sequencing (Extended Data Fig. S1).

\section{Table 1 Taxonomic differences in the oral microbiota of HV and GC.}


Taxa (class; order; family; genus; species)

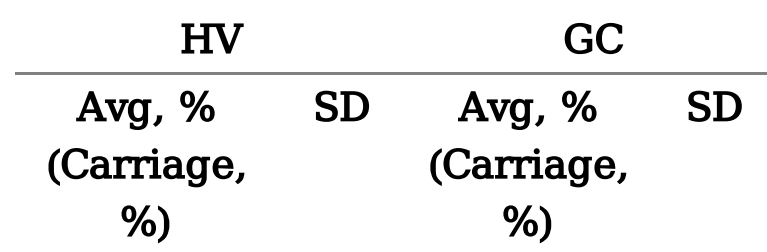

\section{Phylum _ Bacteroidetes}

C__Bacteroidia; o__Bacteroidales;

$f_{-}$Prevotellaceae; $g_{--}$Alloprevotella

c__Bacteroidia; o__Bacteroidales;

$f_{--}$Prevotellaceae; g__Alloprevotella;

s_sp._oral_taxon_308

\begin{tabular}{lllll} 
& \multicolumn{1}{c}{1.85} \\
$0.84(100)$ & 0.81 & $(100)$ & 1.28 & 0.032
\end{tabular}

C__Bacteroidia; o__Bacteroidales;

$f_{-}$Prevotellaceae; $g_{-}$Prevotella;

s_sp._oral_taxon_396

c__Flavobacteriia; o_fFlavobacteriales;

$f_{-}$Flavobacteriaceae; $g_{-}$Bergeyella

$\begin{array}{llllll} & & 0.66 \\ & & & & & \\ (100) & 0.16 & (100) & 0.48 & 0.019\end{array}$

\section{Phylum _ Firmicutes;}

C__Bacilli; o__Lactobacillales;

f_Carnobacteriaceae

c__Bacilli; o__Lactobacillales;

f_Carnobacteriaceae; $g_{-}$_Granulicatella

c__Negativicutes; o__Selenomonadales;

$f_{--}$Veillonellaceae; g__Megasphaera

c__Bacilli; o__Lactobacillales;

$f_{--}$Streptococcaceae; $g_{--}$Streptococcus;

s__salivarius

C__Negativicutes; o__Selenomonadales;

$f_{--}$Veillonellaceae; $g_{--}$Megasphaera;

$s_{-}$micronuciformis

$\begin{array}{cccccc}0.05(92) & 0.04 & \begin{array}{c}0.12 \\ (100) \\ 0.11\end{array} & 0.11 & 0.046 \\ 0.42(100) & 0.47 & (100) & 0.10 & 0.047\end{array}$

Phylum _ Fusobacteria;

C__Fusobacteriia; o__Fusobacteriales;

$f_{-}$Leptotrichiaceae; $g_{-}$Leptotrichia;

s_sp._oral_taxon_392

$\begin{array}{lllll}2.89(100) & 2.24 & \begin{array}{c}1.39 \\ (100) \\ 1.39\end{array} & 0.85 & 0.048 \\ 2.89(100) & 2.24 & \begin{array}{l}(100) \\ 0.15\end{array} & 0.85 & 0.048 \\ 0.03(75) & 0.04 & (100) & 0.17 & 0.033\end{array}$

\section{Phylum _ Proteobacteria}

C__Gammaproteobacteria; o__Pasteurellales;

$f_{--}$Pasteurellaceae; g__Aggregatibacter;

s_segnis

$0.10(\mathbf{1 0 0 )} \quad 0.14 \quad \mathbf{0 . 4 1}(\mathbf{7 5 )} \quad 0.44 \quad 0.032$

\section{Phylum_Saccharibacteria_(TM7)}

C_-TM7_[C-1]; o_-TM7_[O-1]; $f_{--} T M 7 \_[F-1]$;

g_tM7_[G-6]

C_-TM7_[C-1]; o_-TM7_[O-1]; $f_{--} T M 7 \_[F-1]$;

$\begin{array}{lllll}0.27(100) & 0.21 & \text { (100) } & 0.08 & 0.007\end{array}$

g_tTM7_[G-6]; s__sp._oral_taxon_870

$0.19(92) \quad 0.16 \quad 0.03(42) \quad 0.05 \quad 0.005$

$\begin{array}{lllll}0.19(92) & 0.16 & 0.03(42) & 0.05 & 0.005\end{array}$ 
These dramatically different species between the GC and HV groups are further analyzed by ROC curves, which indicate that $A$. segnis, S. salivarius, M. micronuciformis and Oral taxon 870 achieve better diagnostic power with an AUC value greater than $0.75(\mathrm{p}<0.001)$ (Extended Data Fig. S2 A-D). Logistic regression is used to evaluate different combinations for distinguishing GC. The combination of $A$. segnis and $S$. salivarius yield an AUC value of $0.98(95 \% \mathrm{Cl} 0.93$ to $1.0, \mathrm{p}<0.001)$ with $100 \%$ sensitivity and $91.7 \%$ specificity for distinguishing the GC patients from the HVs (Extended Data Fig. S2 E). The combination of S. salivarius and Oral taxon 870 also achieved excellent performance with an AUC value of $0.93(95 \% \mathrm{Cl}$ 0.82 to $1.0, p<0.001$ ) for distinguishing the GC patients from the HVs (sensitivity: $91.7 \%$, and specificity: 75\%) (Extended Data Fig. S2 F). These results demonstrate that changes in the oral microbiota are associated with GC and have potential as cancer biomarkers.

Our previous studies demonstrated that GC could induce alterations in salivary protein glycopatterns, especially fucosylated glycoproteins, in the saliva of GC patients ${ }^{13}$. Here, a fucosylated neoglycoprotein (Fuc-BSA) and other neoglycoproteins (Gal-BSA and Man-BSA) are synthesized and their roles in the proliferation and adhesion of $A$. segnis are evaluated (Extended Data Fig. S3). The results showed that the proliferation of $A$. segnis is not affected by $3-100 \mu \mathrm{g} / \mathrm{mL}$ Fuc-BSA, Gal-BSA, Man-BSA, their monosaccharides (Fuc, Gal, and Man) or BSA (data not shown). In addition, 30 and $100 \mu \mathrm{g} / \mathrm{mL}$ Fuc-BSA significantly decreases the adhesion of $A$. segnis to CAL-27 cells (Fig. 3); however, 3 and $10 \mu \mathrm{g} / \mathrm{mL}$ FucBSA and 30 and $100 \mu \mathrm{g} / \mathrm{mL}$ Man-BSA and Gal-BSA not affect this process (Extended Data Fig. S4A and S4B). A similar phenomenon is observed in HOEC cells, and 30 and $100 \mu \mathrm{g} / \mathrm{mL}$ Fuc-BSA also significantly decreases the adhesion of $A$. segnis to HOEC cells. These results demonstrate that the fucose moieties of proteins can disturb $A$. segnis attachment to the cell surface, which implies that they can prevent periodontal disease.

Among these seven bacteria, A. segnis and M. micronuciformis could be cultivated and showed increased abundance in the GC patients. Haemophilus segnis is first isolated in $1977^{14}$ and reclassified into the Aggregatibactergenus in $2006^{15}$; it is a normal commensal in oral humans but is rarely reported as a pathogen. Infective endocarditis is the most well-known infection but is still rarely reported to be associated with $A$. segnis ${ }^{16}$. Periodontal disease, acute appendicitis, pancreatic abscess, and cholecystitis have also been associated with $A$. segnis on occasion ${ }^{17,18}$. Previous studies have focused mainly on case reports, and the true prevalence of this bacterium in human infections is probably underreported; the underlying mechanism associated with diseases is not yet explicitly known. To understand the related mechanisms, the glycopatterns of the outer membrane and total protein of $A$. segnis and $A$. segnis treated with 30 and $100 \mu \mathrm{g} / \mathrm{mL}$ Fuc-BSA are analyzed by lectin microarrays. There are 9 lectins (e.g., Jacalin, GSL-II, and SNA) that show decreased expression levels of the glycopatterns (e.g., GalB1-3GalNAca-Ser/Thr, GalNAca-Ser/Thr and Sia2-6Gal/GalNAc), while there are 3 lectins (PHA-E, $L E L$, and MAL-I) that show extremely increased expression levels of the glycopatterns (Bisecting GlcNAc, high mannose-type N-glycans, and Gal $\beta-1,4 \mathrm{GICNAc}$ ) on the outer membrane of $A$. segnis treated by FucBSA (Extended Data Fig. S5A). However, 4 lectins (PHA-E, PNA, LEL and GSL-I) show increased 
expression levels of glycopatterns (bisecting GlcNAc, Galß1-3GalNAca-Ser/Thr, high mannose-type Nglycans, and aGalNAc/aGal) in the total protein of $A$. segnis treated with Fuc-BSA (Extended Data Fig. S5B). These results implied that Fuc-BSA mainly affects glycopatterns on the outer membrane of $A$. segnis. Annotation of $A$. segnis in the carbohydrate-active enzymes database (CAZy) revealed that 24 putative glycosyltransferases (GTs) are distributed among 11 glycosyltransferase families. Of these, 8 GTs are associated with lipopolysaccharide (LPS) biosynthesis, 7 GTs are associated with cell wall formation, and others are associated with glycosylation, glycogen synthase, and carbohydrate metabolism (Extended Data Table S2) ${ }^{19}$. The qPCR results show that 3 mRNAs of LPS-associated GTs (NCTC10977_00605 (Rfaq_1), NCTC10977_00729, and NCTC10977_00649 (WaaA)) are significantly decreased and that the mRNA of mannosyltransferase $\mathrm{OCH} 1$ is significantly increased in $A$. segnis treated with Fuc-BSA (Fig. S7A and S7B), which indicate that Fuc-BSA interfered with the biosynthesis of core oligosaccharides and 0 -antigenic polysaccharides of LPS. These results suggest that Fuc-BSA mainly altered the glycopatterns of the outer membrane of $A$. segnis.

Second, Candida albicans (C. albicans) is the most prevalent human fungal pathogen causing infections and oral diseases in T2DM patients ${ }^{20,21}$. Our previous work showed that the expression level of the SAa23 structure in the saliva of T2DM patients is significantly lower than that of healthy people, which is related to T2DM susceptibility to avian influenza virus ${ }^{10}$. Here, sialoglycoproteins from bovine milk are used to inhibit the growth and adhesion of $C$. albicans ${ }^{22}$. The results show that their growth can be inhibited gradually by different concentrations $(50,100,200$, and $400 \mu \mathrm{g} / \mathrm{mL}$ ) of sialoglycoproteins (Fig. 4A) and be inhibited entirely by $400 \mu \mathrm{g} / \mathrm{mL}$ sialoglycoproteins with an IC50 of $142.8 \mu \mathrm{g} / \mathrm{mL}$ (Fig. 4B). To determine the roles of Siaa2-3/6Gal glycans, $400 \mu \mathrm{g} / \mathrm{mL}$ desialoglycoproteins (sialoglycoproteins treated with sodium periodate), sialoglycoproteins without Siaa2-3Gal (sialoglycoproteins treated with a2-3 neuraminidase S), sialic acid, and BSA are also used to inhibit the growth of $C$. albicans. The results show that they cannot inhibit the growth of $C$. albicans, which implies that the Siaa2-3 moieties of sialoglycoproteins play a major role against $C$. albicans. Furthermore, $200 \mu \mathrm{g} / \mathrm{mL}$ sialoglycoproteins and desialoglycoproteins are used to blockade $C$. albicans to adhere to CAL-27 cells. Notably, sialoglycoproteins significantly inhibit the adhesion of $C$. albicans to CAL-27 cells; however, desialoglycoproteins cannot inhibit this process. These results also demonstrate that the Siaa2-3Gal moieties of sialoglycoproteins play a major role in inhibiting the adhesion of $C$. albicans to CAL-27 cells (Fig. 4D, E).

Currently, there are several processes that underlie the transition of a microbial community to a state of dysbiosis. In particular, diet can affect the community composition, and overexposure to dietary carbohydrates and host factors promotes the production of extracellular polymeric substances (EPS) and acidic metabolites, which cause the accumulation of acidogenic and aciduric microorganisms, thus driving the transition to a pathogenic biofilm community ${ }^{23-25}$. Bacteria can obtain nutrients from glycoproteins in saliva and gingival crevicular fluid during fasting ${ }^{26,27}$. Glycoproteins can be broken down into sugars and proteins and metabolized by bacteria that can gain energy to survive. During metabolism, sugars and proteins are further converted into acidic and basic small molecules, which can neutralize 
each other to maintain a neutral state in the mouth. If sugar consumption is low and infrequent, the microbial communities on teeth remain stable. Despite being able to produce acids that demineralize enamel, the episodic $\mathrm{pH}$ decrease can be readily neutralized by saliva, which restores and maintains the mineralization of enamel ${ }^{28}$. However, once a community has transitioned to a dysbiotic state, the structural stability of functionally specialized components will allow the condition to persist for an extended period of time, promoting the development of oral diseases such as periodontitis and dental caries $^{29-31}$.

However, our studies demonstrated that human disease could induce different alterations in salivary protein glycopatterns ${ }^{6}$. The altered salivary protein glycopatterns (e.g., fucosylated or sialylated structures) induced by GC or T2DM are also drivers of dysbiosis of oral microbial communities and ultimately dental caries and periodontitis. The fucosylated neoglycoproteins and SAa2-3Gal structure can inhibit the growth and adhesion of $A$. segnis and $C$. albicans from the oral cavity of GC and T2DM, respectively. These findings provide a novel theory that dynamic communities of oral microbiota are regulated naturally by host salivary protein glycopatterns, having important implications for developing new carbohydrate drugs for oral and body health.

\section{Methods}

\section{Study Population}

The collection and use of human saliva samples for the research presented here were approved by the Ethical Committee of Northwest University (Xi'an, China), First Affiliated Hospital of Xi'an Jiaotong University (Xi'an, China). Written informed consent was received from participants for the collection of their saliva samples. This study was conducted in accordance with the ethical guidelines of the Declaration of Helsinki. After a standardized endoscopic procedure and histopathological evaluation, individuals who were diagnosed with $\mathrm{GC}(\mathrm{N}=12)$ were enrolled in this study. The age- and sex-matched $\mathrm{HVs}(\mathrm{N}=12)$ were free of any precancerous lesions, as confirmed by gastroscopy. All the participants were $\geq 40$ years old, and no significant differences were observed between the two groups regarding demographic, socioeconomic, and lifestyle characteristics. Patients who received preoperative radiotherapy, chemotherapy, chemoradiotherapy or antibiotic therapy were excluded from the study. The clinical characteristics of the HVs and GC patients are summarized in Extended Data Table S3.

\section{Sequencing and Data Analysis}

Bacterial genomic DNA was extracted by a Bacterial DNA Isolation Kit D3350-01 (OMEGA, USA) following the manufacturer's instructions. The $16 \mathrm{~S}$ rDNA V3-V4 regions were amplified by specific bacterial primers 341F-806R with barcodes 341F: CCTAYGGGRBGCASCAG, and 806R: GGACTACNNGGGTATCTAAT. All PCRs were carried out with Phusion High-Fidelity PCR Master Mix (New England Biolabs, USA). The PCR products were extracted and quantified, pooled in equimolar concentrations and sequenced using the lon $\mathrm{S} 5^{\mathrm{TM}} \mathrm{XL}$ platform according to the manufacturer's recommendations. The methods for sequence 
screening, diversity analysis and taxonomy-based analysis were performed as described in previous reports $^{32}$. Differences between two arbitrary data sets were tested by the Wilcoxon test for each species using SPSS statistics 20.

\section{Synthesis of neoglycoproteins}

The synthesis of neoglycoprotein is divided into three steps (Extended Data Fig. S6A): the monosaccharides coupled with the linker (5-(4-hydroxyphenyl)pentanoic acid) to form glycan conjugates, and the carboxylates $(-\mathrm{COOH})$ from glycan conjugates reacted to NHS in the presence of EDC, resulting in a semistable NHS, followed by reaction with amines (-NH2) of BSA to form amide crosslinks. Compared with the band of identically processed BSA, the upward migration bands of neoglycoproteins are obviously observed in the SDS-PAGE results (Extended Data Fig. S6B).

First, $5 \mu \mathrm{mol}$ of each monosaccharide (fucose, mannose, and galactose (Sigma-Aldrich, USA)) was mixed with $2.5 \mu \mathrm{mol}$ of 5-(4-hydroxyphenyl) pentanoic acid, dissolved in N,N-dimethylformamide (DMF), decreased to a pH under 3.0 using sulfuric acid, and then incubated at $60^{\circ} \mathrm{C}$ for $12 \mathrm{~h}$ under gentle shaking with an appropriate amount of silicon dioxide. Second, after chloroform extraction, the glycan complexes were mixed with $10 \mu \mathrm{mol}$ EDC and $25 \mu \mathrm{mol}$ NHS in activation buffer ( $0.1 \mathrm{M}$ MES (2[morpholino] ethanesulfonic acid), $0.5 \mathrm{M} \mathrm{NaCl}, \mathrm{pH} 6.0$ ) and incubated at room temperature for $4 \mathrm{~h}$. Third, the products were added to $100 \mathrm{nmol}$ BSA solution (BSA dissolved in PBS), and then the buffer $\mathrm{pH}$ was adjusted to 7.4 and incubated at room temperature for $4 \mathrm{~h}$. The Quench reaction was performed by adding hydroxylamine to a final concentration of $10 \mathrm{mM}$. The synthesized neoglycoproteins were dialyzed against PBS overnight and concentrated using an Amicon ultra-4 $30 \mathrm{kDa}$ ultrafiltration unit (Millipore, USA). The neoglycoprotein solutions were collected, filtered through $0.22 \mu \mathrm{m}$ filters and stored at $-80^{\circ} \mathrm{C}$. The synthesized neoglycoproteins were analyzed by $10 \%$ SDS-PAGE and then stained directly with alkaline silver.

\section{Lectin Microarrays and Data Analysis}

A lectin microarray was produced using 37 lectins with different binding preferences covering $\mathrm{N}$ - and $\mathrm{O}$ linked glycans ${ }^{9}$. The Cy3-labeled glycoproteins or bacteria were incubated on a lectin microarray at $37^{\circ} \mathrm{C}$ for $3 \mathrm{~h}$ with gentle rotation in the dark. The slides were washed three times with PBST and PBS, centrifuged dry and scanned immediately with $70 \%$ photomultiplier tubes and $100 \%$ laser power settings using a Genepix 4000B confocal scanner (Axon Instruments, USA). The acquired images were analyzed at $532 \mathrm{~nm}$ for Cy3 detection by Genepix 3.0 software. Differences between two arbitrary data sets or multiple data sets were tested by Wilcoxon test or one-way ANOVA for each lectin signal using SPSS statistics 20 .

\section{Real-Time PCR (qRT-PCR)}

Total RNA was extracted using the Bacteria Total RNA Isolation Kit (Sangon Biotech, China) and converted into cDNA using the PrimeScript RT reagent Kit (TaKaRa, Japan). The cDNAs were then 
subjected to real-time quantitative polymerase chain reaction (qRT-PCR) analysis ( $A B I$ ViiA ${ }^{T M}$ 7, Bio-Rad, USA) using gene-specific primers (Extended Data Table S4) and TB Green Fast qPCR Mix (TaKaRa, Japan). The $16 \mathrm{~S}$ rRNA gene was used as an endogenous control for normalizing the relative expression of target genes.

\section{Isolation of Sialoglycoproteins from Bovine Milk}

According to the protocol in the literature ${ }^{22}$. In brief, serotonin-functionalized magnetic particle conjugates were thoroughly washed with binding buffer $\left(20 \mathrm{mM} \mathrm{Na}_{3} \mathrm{PO}_{4}, \mathrm{pH} 6.0\right)$ and incubated sequentially with $1 \mathrm{~mL}$ of binding buffer containing $0.2 \mathrm{~mL}$ bovine milk with gentle shaking at room temperature for $3 \mathrm{~h}$ without light. The unbound proteins were removed from the conjugates with binding buffer until there were no detectable proteins. Finally, the sialoglycoproteins were eluted from the conjugates with eluting buffer $\left(0.1 \mathrm{M} \mathrm{NaHCO}_{3}, 0.5 \mathrm{M} \mathrm{NaCl}, \mathrm{pH} 8.3\right)$ and quantitated with the BCA method.

\section{Destruction of Siaa2-3/6Gal Moieties and Enzymolysis of Siaa2-3 Gal Moieties of the Isolated Sialoglycoproteins}

The Siaa2-3/6Gal structures of the isolated sialoglycoproteins were destroyed by treatment with $1 \mathrm{mM}$ sodium periodate at $0{ }^{\circ} \mathrm{C}$ for $15 \mathrm{~min}$, as described previously ${ }^{33}$. Then, sodium periodate was removed by using an ultracentrifugal filter (Ultracel-3k, Millipore Corp., Billerica, MA). The Siaa2-3 Gal moieties of the isolated sialoglycoproteins were digested by a2-3 neuraminidase S (New England Biolabs, USA) according to the manufacturer's recommendations.

\section{Bacterial Strains and Cell Culture}

A. segnis was obtained from the American Type Culture Collection (ATCC) and grown in 814 GC agar/broth medium supplemented with $15 \mu \mathrm{g} / \mathrm{mL} \mathrm{NAD}^{+}$(Solarbio, Beijing, China) and $30 \mu \mathrm{g} / \mathrm{mL}$ vancomycin (Solarbio, Beijing, China) at $37^{\circ} \mathrm{C}$ with $5 \% \mathrm{CO}^{2}$. C. albicans ATCC 10231 was obtained from the National Center for Medical Culture Collections (CMCC) and aerobically grown at $37^{\circ} \mathrm{C}$ in Sabouraud's dextrose broth supplemented with $50 \mathrm{mg} / \mathrm{ml}$ gentamicin (Solarbio, Beijing, China). CAL-27 and HOEC cells were obtained from Otwo Biotech Inc. (Shenzhen, China), cultured in DMEM (HyClone, Waltham, MA, USA) supplemented with $10 \%$ FBS (Gibco, Grand Island, NY, USA) and maintained at $37^{\circ} \mathrm{C}$ with $5 \% \mathrm{CO}^{2}$.

\section{Adhesion Assays}

A. segnis or $C$. albicans were washed with fresh medium three times, centrifuged and diluted with fresh medium containing $100 \mu \mathrm{M}$ FITC-d-Lys (Xiamen Bioluminor Bio-Technology, Xiamen, China). After 30 min of incubation at $37^{\circ} \mathrm{C}$, the cells were centrifuged, washed with fresh medium three times, and then resuspended in PBS. CAL-27 or HOEC cells $\left(1 \times 10^{5}\right.$ cells) were inoculated into confocal culture dishes (JingAn Biotechnology, Shanghai, China) and cultured in complete medium. Cells were grown to $60-70 \%$ confluence and then synchronized by serum starvation overnight. One hundred microliters of serum-free 
DMEM with $5 \mu \mathrm{M}$ DiD (AAT Bioquest, USA) was pipetted onto the dishes and gently agitated until all the cells were covered. After 30 min of incubation at $37^{\circ} \mathrm{C}$, the cells were washed three times with DMEM. $C$.

albicans or FITC-labeled $A$. segnis $\left(1 \times 10^{6}\right.$ cells $\left./ \mathrm{mL}\right)$ was added to the dishes in $500 \mu \mathrm{L}$ serum-free DMEM, which was allowed to infect HOEC or CAL-27 cells for $45 \mathrm{~min}$ for adhesion. The infected cells were washed three times with $1 \times$ PBS and immobilized by incubating with $0.2 \%$ Triton X-100 in $4 \%$ paraformaldehyde for 30 min at RT. After DAPI staining (Thermo Fisher Scientific, Waltham, USA), a laser scanning confocal microscope FV 1000 (Olympus, Tokyo, JPN) was used to acquire the images with the merged channels of FITC $(\operatorname{Ex} / \operatorname{Em}(\mathrm{nm})=488 / 520)$, DiD $(\operatorname{Ex} / \operatorname{Em}(\mathrm{nm})=650 / 670)$ and DAPI $(E x / E m$ $(n m)=358 / 461)$. A fluorescence microscope (Olympus, Tokyo, JPN) was used to observe the adhesion of C. albicans to cells.

\section{References}

1 Lamont, R. J., Koo, H. \& Hajishengallis, G. The oral microbiota: dynamic communities and host interactions. Nat Rev Microbio/ 16, 745-759, doi:10.1038/s41579-018-0089-x (2018).

2 Fan, X. et al. Human oral microbiome and prospective risk for pancreatic cancer: a population-based nested case-control study. Gut 67, 120-127, doi:10.1136/gutjnl-2016-312580 (2018).

3 Flemer, B. et al. The oral microbiota in colorectal cancer is distinctive and predictive. Gut $67,1454-$ 1463, doi:10.1136/gutjnl-2017-314814 (2018).

4 Long, J. et al. Association of oral microbiome with type 2 diabetes risk. J Periodontal Res 52, 636-643, doi:10.1111/jre.12432 (2017).

5 Peters, B. A. et al. Oral Microbiome Composition Reflects Prospective Risk for Esophageal Cancers. Cancer research 77, 6777-6787, doi:10.1158/0008-5472.Can-17-1296 (2017).

6 Zhang, J. et al. Identification of abnormal fucosylated-glycans recognized by LTL in saliva of HBVinduced chronic hepatitis, cirrhosis, and hepatocellular carcinoma. Glycobiology 29, 242-259, doi:10.1093/glycob/cwy108 (2019).

7 Escapa, I. F. et al. New Insights into Human Nostril Microbiome from the Expanded Human Oral Microbiome Database (eHOMD): a Resource for the Microbiome of the Human Aerodigestive Tract. mSystems 3, doi:10.1128/mSystems.00187-18 (2018).

8 Belda-Ferre, P. et al. The oral metagenome in health and disease. The ISME journa/ 6, 46-56, doi:10.1038/ismej.2011.85 (2012).

9 Qin, Y. et al. Age- and sex-associated differences in the glycopatterns of human salivary glycoproteins and their roles against influenza A virus. Journal of proteome research 12, 2742-2754, doi:10.1021/pr400096w (2013). 
10 Zhong, Y. et al. Avian Influenza Virus Infection Risk in Humans with Chronic Diseases. Scientific reports 5, 8971 (2015).

11 Shu, J., Yu, H., Li, X., Zhang, D. \& Liu, X. Salivary glycopatterns as potential biomarkers for diagnosis of gastric cancer. Oncotarget 8, 35718-35727 (2017).

12 Liu, X. et al. Salivary Glycopatterns as Potential Biomarkers for Screening of Early-Stage Breast Cancer. EBioMedicine, 70-79 (2018).

$13 \mathrm{Shu}, \mathrm{J}$. et al. Identification of $\mathrm{N}$ - and O-linked glycans recognized by AAL in saliva of patients with atrophic gastritis and gastric cancer. Cancer Biomarkers 22, 669-681 (2018).

14 Kilian, M. A taxonomic study of the genus Haemophilus, with the proposal of a new species. J Gen Microbio/ 93, 9-62, doi:10.1099/00221287-93-1-9 (1976).

15 Nørskov-Lauritsen, N. \& Kilian, M. Reclassification of Actinobacillus actinomycetemcomitans, Haemophilus aphrophilus, Haemophilus paraphrophilus and Haemophilus segnis as Aggregatibacter actinomycetemcomitans gen. nov., comb. nov., Aggregatibacter aphrophilus comb. nov. and Aggregatibacter segnis comb. nov., and emended description of Aggregatibacter aphrophilus to include $\mathrm{V}$ factor-dependent and V factor-independent isolates. Int J Syst Evol Microbio/ 56, 2135-2146, doi:10.1099/ijs.0.64207-0 (2006).

16 Revest, M., Egmann, G., Cattoir, V. \& Tattevin, P. HACEK endocarditis: state-of-the-art. Expert Rev Anti Infect Ther 14, 523-530, doi:10.1586/14787210.2016.1164032 (2016).

17 Welch, W. D., Southern, P. M., Jr. \& Schneider, N. R. Five cases of Haemophilus segnis appendicitis. J Clin Microbio/ 24, 851-852, doi:10.1128/jcm.24.5.851-852.1986 (1986).

18 Carson, H. J., Rezmer, S. \& Belli, J. Haemophilus segnis cholecystitis: a case report and literature review. The Journal of infection 35, 85-86, doi:10.1016/s0163-4453(97)91193-2 (1997).

19 Whitfield, C. \& Trent, M. S. Biosynthesis and export of bacterial lipopolysaccharides. Annu Rev Biochem 83, 99-128, doi:10.1146/annurev-biochem-060713-035600 (2014).

20 Harriott, M. M. \& Noverr, M. C. Importance of Candida-bacterial polymicrobial biofilms in disease. Trends in microbiology 19, 557-563, doi:10.1016/j.tim.2011.07.004 (2011).

21 Casarin, R. C. et al. Subgingival biodiversity in subjects with uncontrolled type-2 diabetes and chronic periodontitis. J Periodontal Res 48, 30-36, doi:10.1111/j.1600-0765.2012.01498.x (2013).

22 Wang, X. et al. Purification of sialoglycoproteins from bovine milk using serotonin-functionalized magnetic particles and their application against influenza A virus. Food \& Function 11, 6911-6920, doi:10.1039/D0FO01447H (2020). 
23 Bowen, W. H., Burne, R. A., Wu, H. \& Koo, H. Oral Biofilms: Pathogens, Matrix, and Polymicrobial Interactions in Microenvironments. Trends in microbiology 26, 229-242, doi:10.1016/j.tim.2017.09.008 (2018).

24 Marsh, P. D. \& Zaura, E. Dental biofilm: ecological interactions in health and disease. Journal of clinical periodontology 44 Suppl 18, S12-s22, doi:10.1111/jcpe.12679 (2017).

25 Takahashi, N. \& Nyvad, B. The role of bacteria in the caries process: ecological perspectives. Journal of dental research 90, 294-303, doi:10.1177/0022034510379602 (2011).

26 Dabdoub, S. M., Ganesan, S. M. \& Kumar, P. S. Comparative metagenomics reveals taxonomically idiosyncratic yet functionally congruent communities in periodontitis. Scientific reports 6, 38993, doi:10.1038/srep38993 (2016).

27 Lu, M., Xuan, S. \& Wang, Z. Oral microbiota: A new view of body health. Food Science and Human Wellness 8, 8-15, doi:https://doi.org/10.1016/j.fshw.2018.12.001 (2019).

28 Tanner, A. C. R., Kressirer, C. A., Rothmiller, S., Johansson, I. \& Chalmers, N. I. The Caries Microbiome: Implications for Reversing Dysbiosis. Advances in dental research 29, 78-85, doi:10.1177/0022034517736496 (2018).

29 Mark Welch, J. L., Rossetti, B. J., Rieken, C. W., Dewhirst, F. E. \& Borisy, G. G. Biogeography of a human oral microbiome at the micron scale. Proc Natl Acad Sci U S A 113, E791-800, doi:10.1073/pnas.1522149113 (2016).

30 Suwannakul, S., Stafford, G. P., Whawell, S. A. \& Douglas, C. W. I. Identification of bistable populations of Porphyromonas gingivalis that differ in epithelial cell invasion. Microbiology (Reading, England) 156, 3052-3064, doi:10.1099/mic.0.038075-0 (2010).

31 Valm, A. M. et al. Systems-level analysis of microbial community organization through combinatorial labeling and spectral imaging. Proc Natl Acad Sci U S A 108, 4152-4157, doi:10.1073/pnas.1101134108 (2011).

32 Yang, K. et al. Microbial diversity in combined UAF-UBAF system with novel sludge and coal cinder ceramic fillers for tetracycline wastewater treatment. Chemical Engineering Journal 285, 319-330, doi:https://doi.org/10.1016/j.cej.2015.10.019 (2016).

$33 \mathrm{Kim}, \mathrm{D}$. S. et al. Both a2,3- and a2,6-linked sialic acids on O-linked glycoproteins act as functional receptors for porcine Sapovirus. PLoS Pathog 10, e1004172, doi:10.1371/journal.ppat.1004172 (2014).

\section{Figures}


A

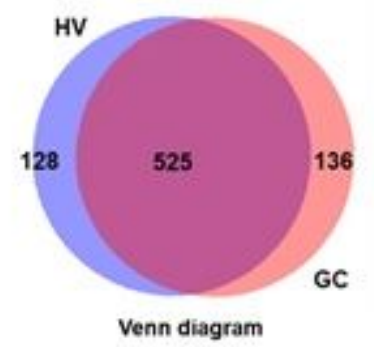

B

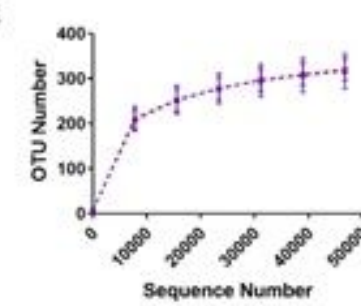

D
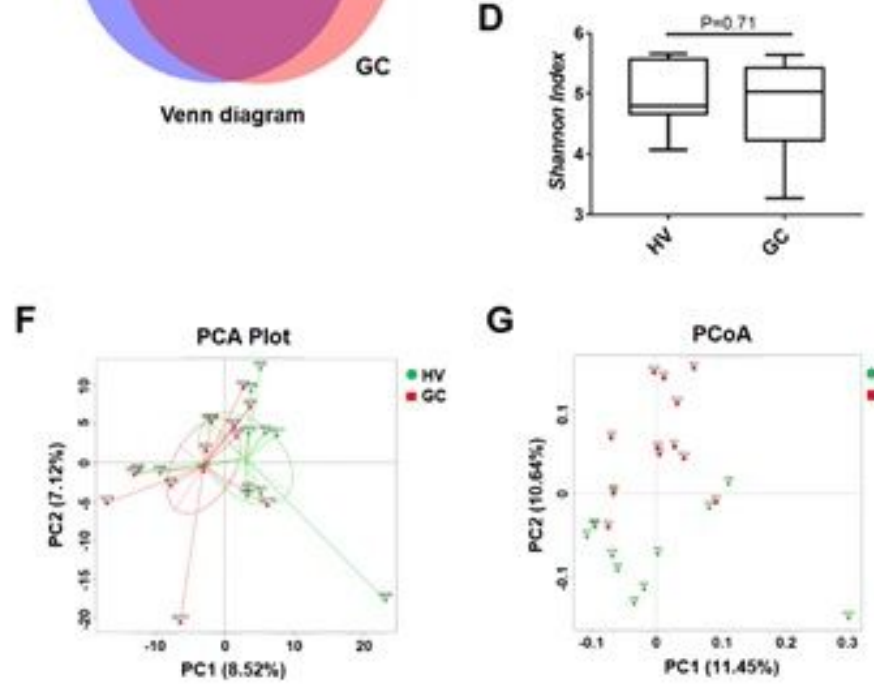

G

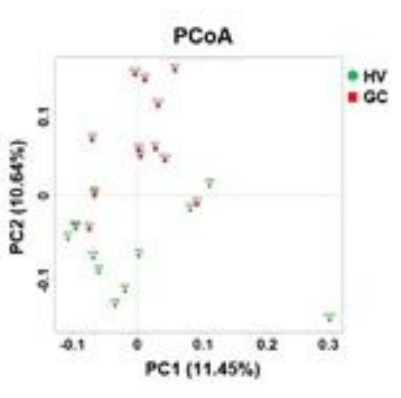

C

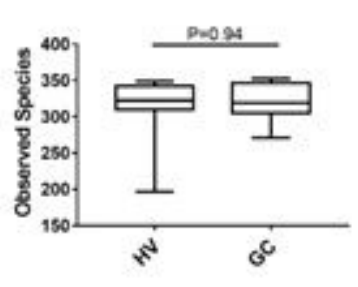

E
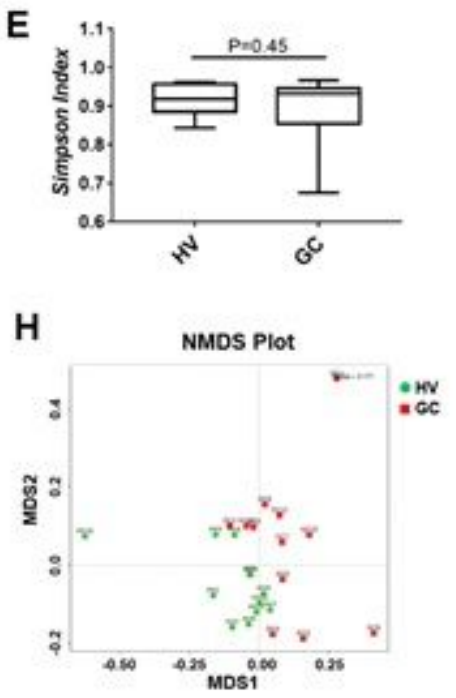

Figure 1

Comparison of the structures of the oral microbiota between the HV and GC groups. (A) Venn diagram illustrating the overlap of the species identified in the oral microbiota of the HV and GC groups, (B) OTU numbers, (C) Observed species, (D) Shannon index, (E) Simpson index, (F) PCA, (G) PCoA, (H) NMDS analysis. 


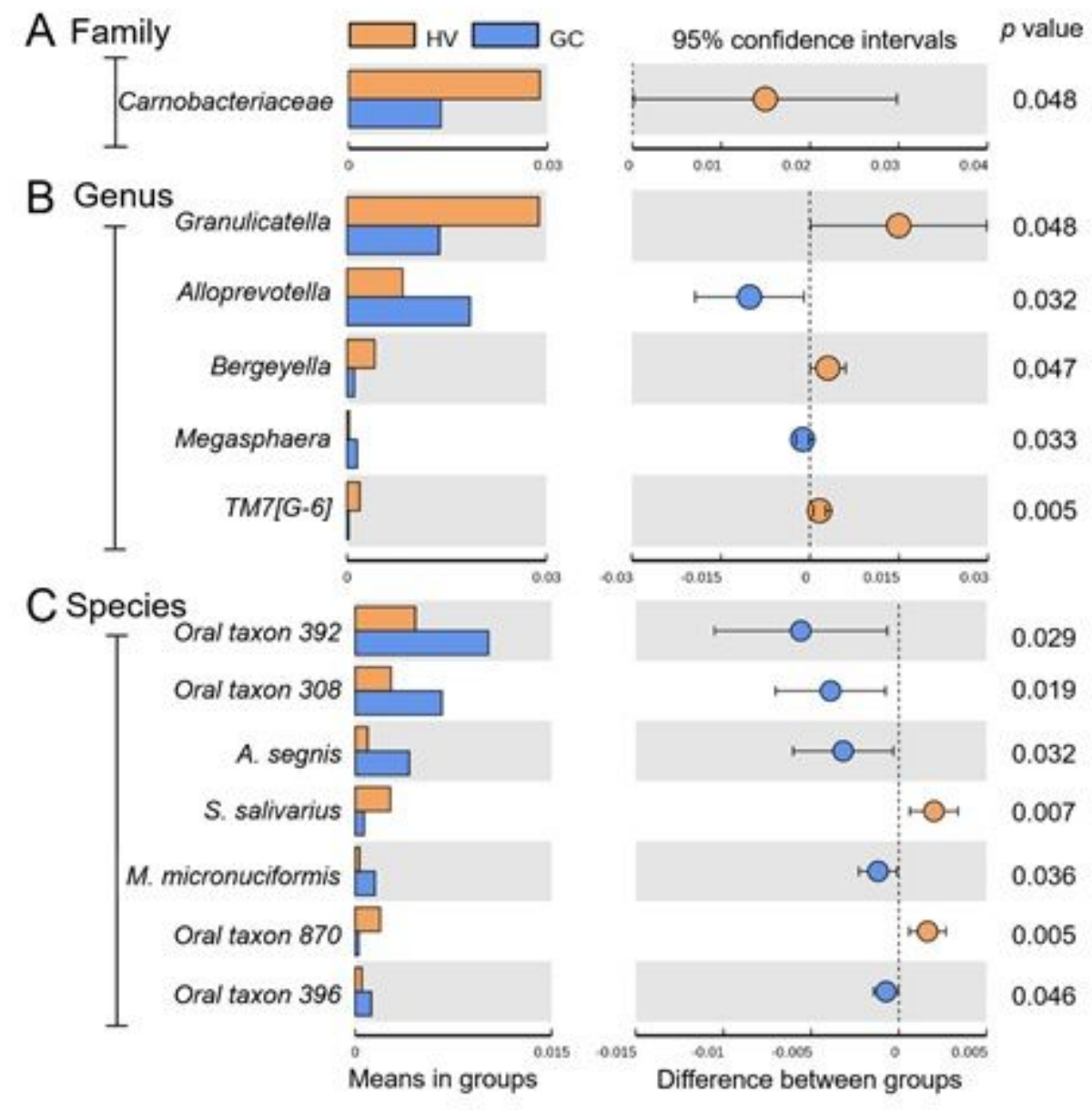

\section{Figure 2}

Comparison of the relative abundance at the bacterial family (A), genus (B) and species (C) levels in the HV and GC groups. 


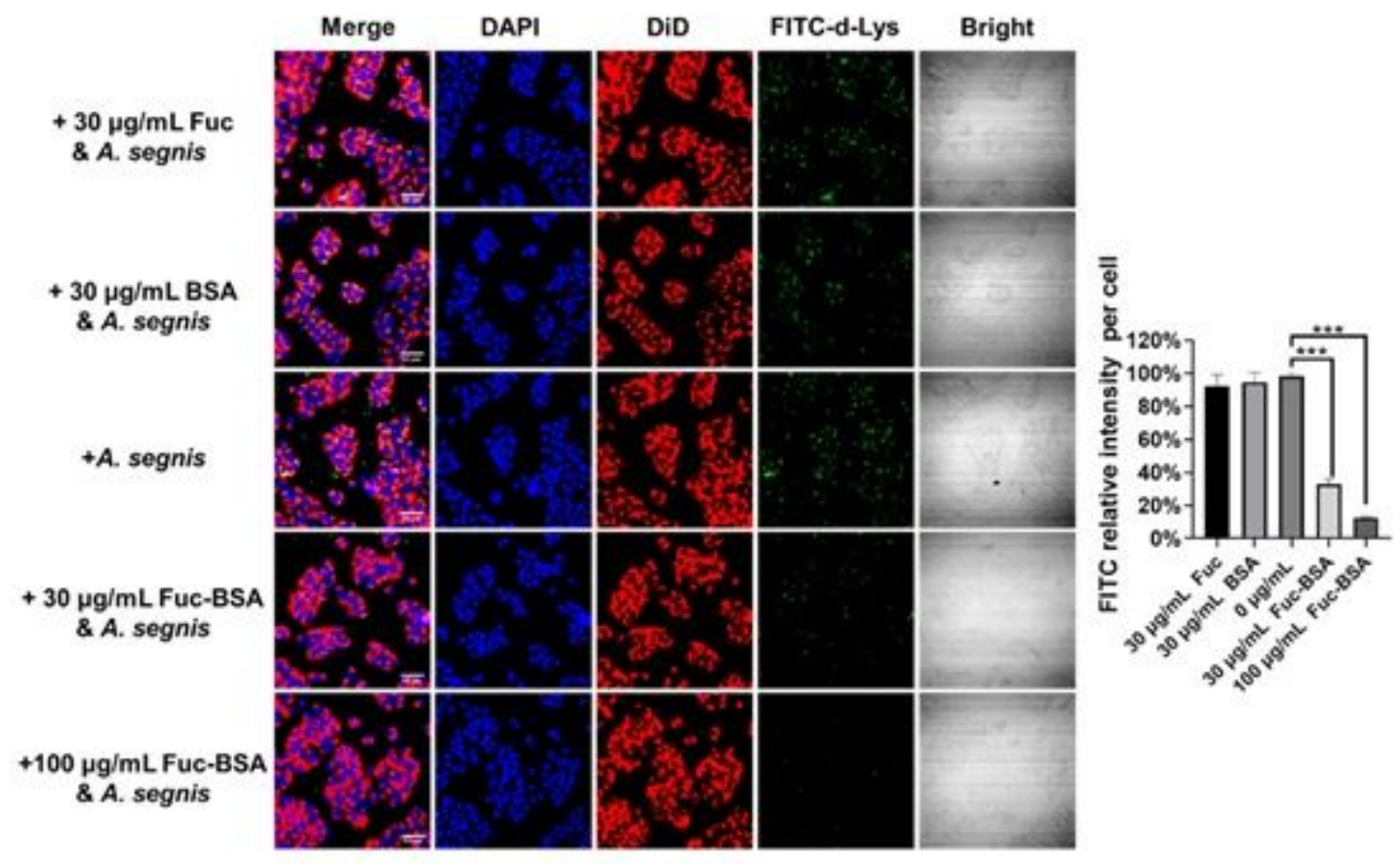

Figure 3

The adhesion of A. segnis to HOEC cells. A. segnis is added to HOEC cells with Fuc-BSA, BSA or Fuc and allowed to infect for $90 \mathrm{~min}$. Fuc-BSA (30 and $100 \mu \mathrm{g} / \mathrm{mL}$ ) significantly decreased the adhesion of $A$. segnis to HOEC cells. The images are acquired under the same conditions for the merged channels of FITC $(\operatorname{Ex} / \operatorname{Em}(n m)=488 / 520)$, DiD $(E x / E m(n m)=650 / 670)$ and DAPI $(E x / E m(n m)=358 / 461)$. Statistical significance analysis for FITC fluorescence intensity per cell is computed by one-way ANOVA $(*: p<0.05$, $\star *: p<0.01$, and $* \star *: p<0.001)$. 

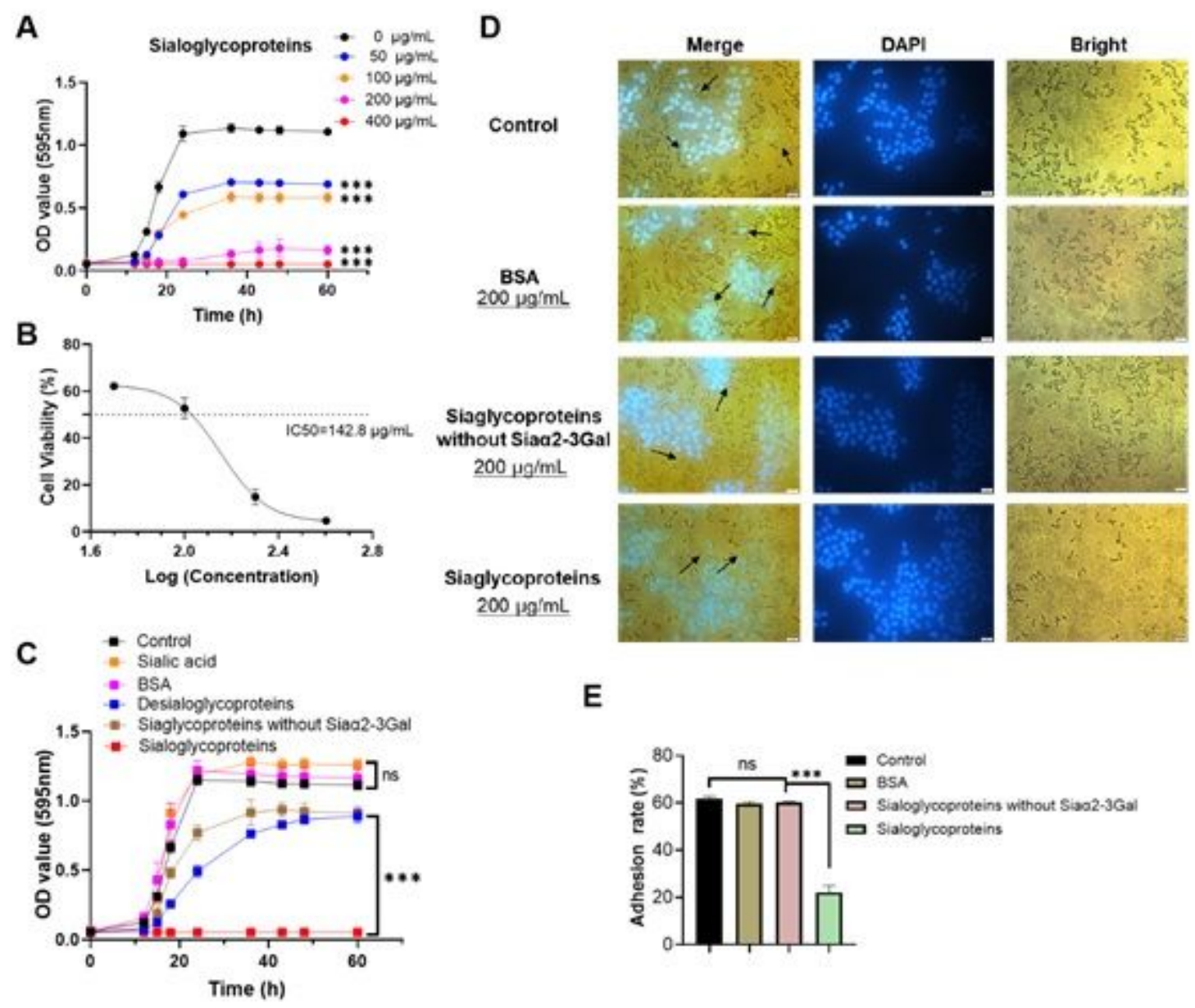

\section{Figure 4}

The growth and adhesion of C. albicans are affected by Sia2-3Gal moieties. (A) Effects of different concentrations of sialoglycoproteins on the growth of C. albicans. (B) The IC50 of sialoglycoproteins against C. albicans. (C) The effects of sialic acid, desialoglycoproteins and sialoglycoproteins without Siaa2-3Gal on the growth of C. albicans. Destruction of Siaa2-3 moieties significantly affect the inhibition activity of sialoglycoproteins against $\mathrm{C}$. albicans, indicating that the Siaa2-3 moieties play a major role in the inhibition progress. (D) The effects of sialoglycoproteins and sialoglycoproteins without Siaa2-3Gal limitation on the adhesion of C. albicans to CAL-27 cells. (E) The adhesion rate of C. albicans to CAL-27 cells.

\section{Supplementary Files}

This is a list of supplementary files associated with this preprint. Click to download.

- ExtendedDataTableS1.rtf

- ExtendedDataTables2.rtf

- ExtendedDataTables3.rtf 
- ExtendedDataTableS4.rtf

- ExtendedDataFigures.pdf 